\title{
Prognostic value of magnetic resonance imaging combined with electromyography in the surgical management of cervical spondylotic myelopathy
}

\author{
FA-JING LIU*, YA-PENG SUN**, YONG SHEN, WEN-YUAN DING and LIN-FENG WANG \\ Department of Spine Surgery, The Third Hospital of Hebei Medical University, Shijiazhuang 050051, P.R. China
}

Received November 10, 2012; Accepted January 14, 2013

DOI: $10.3892 /$ etm.2013.934

\begin{abstract}
The present study aimed to evaluate the value of pre-operative magnetic resonance imaging (MRI) combined with electromyography (EMG) for predicting clinical outcome following surgical management of cervical spondylotic myelopathy (CSM). A total of 94 patients with cervical compressive myelopathy were prospectively enrolled and treated with anterior, posterior and posterior-anterior united decompression between October 2007 and February 2009. Prior to surgery 1.5-T MRI and EMG were performed in all patients. The patients were classified into four types based on the presence (+) or absence (-) of an increased signal intensity (ISI) on the T2-weighted magnetic resonance (MR) images and also based on the positive $(+) /$ negative $(-)$ results of the EMG. The four types were as follows: Type I, MRI/ EMG (-/-); Type II, MRI/EMG (+/-); Type III, MRI/EMG $(-/+)$; and Type IV, MRI/EMG (+/+). The clinical outcome was also graded according to a modified Japanese Orthopedic Association (JOA) scoring system. Furthermore, pre- and post-operative clinical data were statistically analyzed to explore the correlation between the factors. There were 36 cases $(38 \%)$ of Type I, $16(17 \%)$ of Type II, $13(14 \%)$ of Type III and 29 (31\%) of Type IV. According to the analysis of the clinical data between the four types, there were significant differences in the disability classifications, pre-operative JOA scores and disease duration $(\mathrm{P}<0.05)$, but there were no significant differences in gender, age or cord compression ratios $(\mathrm{P}>0.05)$. Until the final follow-up, there was a significant difference in the recovery ratio between the four study groups $(\mathrm{Hc}=27.46, \mathrm{P}<0.05)$. Further comparison showed that the surgical outcome was best in Type I patients and worst in
\end{abstract}

Correspondence to: Dr Yong Shen, Department of Spine Surgery, The Third Hospital of Hebei Medical University, 139 Ziqiang Road, Shijiazhuang 050051, P.R. China

E-mail: shenyong_spine@163.com

*Contributed equally

Key words: cervical spondylotic myelopathy, electromyography, magnetic resonance imaging, prognosis
Type IV patients. In conclusion, there was a distinct correlation between classification and the rate of clinical improvement. Patients who had a negative EMG and those without an ISI on T2-weight images tended to suffer only mild symptoms, a short disease duration and, most significantly, experience a good surgical outcome.

\section{Introduction}

Cervical spondylotic myelopathy (CSM) is a chronic and devastating disease that may be improved by surgical decompression in certain patients. In other patients, surgical intervention does not change their neurological condition or the natural history of the disease. Factors that affect the prognosis and the results of decompressive surgery have been investigated by numerous authors. The increased signal intensity (ISI) of the spinal cord in magentic resonance imaging (MRI) of CSM is thought to reflect the pathological changes in the spinal cord and be indicative of the prognosis (1). However, the significance of intramedullary signal changes in predicting the surgical outcome is greatly controversial $(2,3)$. As it is impossible to quantify the extent of the dysfunction of the spinal cord from physiological findings, electrophysiological findings have been used (4). The purpose of the present study was to elucidate whether changes in the the magnetic resonance (MR) T2 image signal combined with the electromyography (EMG) index reflect the severity of the symptoms and form a prognosis for patients with CSM.

\section{Patients and methods}

Patients. Between October 2007 and February 2009, 94 patients with CSM whose diagnoses were confirmed by clinical and image examination were examined by EMG in the Third Hospital of Hebei and were enrolled in the present study. These examinations were performed to exclude peripheral nerve injuries, including cubital tunnel syndrome and carpal tunnel syndrome. Patients with traumatic cervical cord injuries without bony lesions were also excluded. The group consisted of 56 males and 38 females with a mean age of 52.3 years (range, 46-69 years). The mean disease duration prior to surgery was recorded as 10.5 months (range, 3-32 months). All patients had been evaluated clinically. The patients were diagnosed on the basis of the symptoms and signs of CSM, 
including loss of sensitivity in the hands and/or feet. Several patients had increased reflexes in the knee and ankle. Certain patients presented with stance ataxia and gait unsteadiness in addition to a spastic gait. The patients were treated with anterior, posterior or posterior-anterior united decompression according to their condition. A total of 67 patients were operated on using anterior surgery, 15 patients using posterior surgery and 12 patients using the posterior-anterior combined approach. The study was approved by the HeBei Medical University, Shijiazhuang, China. Written informed patient consent was obtained from the patient.

MRI evaluation. All patients underwent high-resolution MRI with a 1.5-T (Siemens Magnetom Symphony, Malvern, PA, USA) imager prior to surgery. T1- and T2-weighted images of sagittal views of the cervical cord were obtained using a spin echo sequence system for the T1-weighted images and a fast spin echo sequence system for the T2-weighted images. A surface coil was also used. The slice width was $4 \mathrm{~mm}$ and the acquisition matrix was $512 \times 256$. The sequence parameters included a repetition time (TR) of $612 \mathrm{msec} / \mathrm{echo}$ time (TE) of $13 \mathrm{msec}$ for the T1-weighted images and a TR of $2400 \mathrm{msec} / \mathrm{TE}$ of $114 \mathrm{msec}$ for the T2-weighted images. Based on the MRI, the patients were divided into 2 groups, consisting of those patients with (+) and without (-) an ISI on the T2-weighted MR images. These classifications were decided upon by two independent radiologists who were experienced in spinal imaging. The concordance rate between the two observers in evaluating the signal change on the T2-weighted images was $92.1 \%$ and $\kappa$-coefficient was 0.89 . Values of the $\kappa$-coefficient close to 1.0 indicated good reproducibility. The two observers established the final classification by consensus.

EMG evaluation. EMG was performed with a Nicolet Viking IV EMG Unit (Nicolet Biomedical, Madison, WI, USA) prior to surgery. The first dorsal interosseous, extensor digitorum communis, pronator teres, biceps brachii, deltoid, sternocleidomastoideus, brachioradialis and the short abductor thumb muscles were sampled in an assessment of spontaneous activity, motor unit action potential (MUAP) parameters and interference patterns. The motor and sensory nerve conduction velocities of the median, ulnar, radial and axillary nerves were also detected. Using EMG, electrical potential changes of the muscles and the motor and sensory nerve conduction velocities of the peripheral nerves were investigated with aggregate analyses of relaxation, slight constriction and gross constriction, respectively. Presence of a lengthened duration (exceeding the normal value by $20 \%$ in the same age group), large-amplitude MUAPs and polyphasic motor units in at least two myotomes unilaterally or bilaterally were regarded as a positive result $(+)$ due to an anterior horn cell lesion resulting from CSM $(5,6)$. Otherwise, the EMG results were normal (-).

Clinical assessment. Patients were classified into four types according to the results of their MRI and EMG: Type I, MRI/ EMG (-/-); Type II, MRI/EMG (-/+); Type III, MRI/EMG (+/-); and Type IV, MRI/EMG (+/+). The disability classification of CSM depended on a grading system that emphasized gait abnormalities (Table I) (7). The severity of pre-operative
Table I. Disability classification of cervical spondylotic myelopathy (CMS).

\begin{tabular}{ll}
\hline Grade & \multicolumn{1}{c}{ Disability classification } \\
\hline 0 & Root signs and symptoms, no cord involvement \\
1 & Signs of cord involvement, normal gait \\
2 & Mild gait involvement, able to be employed \\
3 & Gait abnormality prevents employment \\
4 & Able to ambulate only with assistance \\
5 & Chairbound or bedridden \\
\hline
\end{tabular}

Table II. Cervical spondylotic myelopathy (CSM) disability distribution in the four MRI/EMG patient types (number of patients).

\begin{tabular}{lrrrrrrr}
\hline & \multicolumn{5}{c}{ Grades of CSM disability } & \\
\cline { 2 - 6 } MRI/EMG & \multicolumn{1}{c}{0} & 1 & 2 & 3 & 4 & 5 & Total \\
\hline$-/-$ & 9 & 10 & 7 & 4 & 3 & 3 & 36 \\
$+/-$ & 3 & 3 & 4 & 3 & 1 & 2 & 16 \\
$-/+$ & 2 & 3 & 2 & 3 & 2 & 1 & 13 \\
$+/+$ & 3 & 3 & 4 & 6 & 8 & 5 & 29 \\
All & 17 & 19 & 17 & 16 & 14 & 11 & 94 \\
\hline
\end{tabular}

Kruskal-Wallis $\mathrm{H}$ test showing $\mathrm{Hc}=9.000, \mathrm{P}=0.029$. MRI, magentic resonance imaging; EMG, elecromyography.

Table III. Surgical outcomes in each EMG/MRI patient type (number of patients).

\begin{tabular}{lrrrrr}
\hline & \multicolumn{4}{c}{ Surgical outcome } \\
\cline { 2 - 4 } MRI/EMG & Excellent & Good & Fair & Poor & Total \\
\hline$-/-$ & 26 & 7 & 2 & 1 & 36 \\
$+/-$ & 7 & 4 & 3 & 2 & 16 \\
$-/+$ & 5 & 3 & 3 & 2 & 13 \\
$+/+$ & 3 & 8 & 12 & 6 & 29 \\
All & 41 & 22 & 20 & 11 & 94 \\
\hline
\end{tabular}

Kruskal-Wallis H test showing $\mathrm{Hc}=27.463, \mathrm{P}<0.001$. MRI, magnetic resonance imaging; EMG, electromyography.

and post-operative neurological deficits was scored according to a modified Japanese Orthopedic Association (JOA) scoring system (highest score, 17 points) (8). To rate the surgical outcome, a recovery rate was calculated using the following formula: Recovery rate $(\%)=$ (post-operative JOA score - pre-operative JOA score)/(17 - pre-operative JOA score) $x$ 100. The surgical outcome was further quantified by categorizing the recovery rate percentage into 4 groups (9): excellent, from 75 to $100 \%$; good, from 50 to $74 \%$; fair, from 25 to $49 \%$; and poor, from 0 to $24 \%$. 
Table IV. Age, disease duration and pre-operative JOA score in each MRI/EMG patient type (mean \pm SD).

\begin{tabular}{|c|c|c|c|c|c|c|}
\hline \multirow[b]{2}{*}{ Factor } & \multicolumn{4}{|c|}{ MRI/EMG } & \multirow{2}{*}{$\begin{array}{c}\text { Test } \\
\text { statistic }\end{array}$} & \multirow[b]{2}{*}{ P-value } \\
\hline & $-/-$ & $+/-$ & $-/+$ & $+/+$ & & \\
\hline Age (years) & $53.17 \pm 5.79$ & $52.32 \pm 13.61$ & $50.60 \pm 7.95$ & $54.43 \pm 10.06$ & $\mathrm{~F}=2.352$ & 0.131 \\
\hline Duration of disease (months) & $7.15 \pm 3.20$ & $10.24 \pm 2.20$ & $9.57 \pm 3.64$ & $16.43 \pm 6.20$ & $\mathrm{~F}=7.830$ & 0.004 \\
\hline Pre-operative JOA score & $10.17 \pm 2.85$ & $9.13 \pm 2.38$ & $9.35 \pm 2.50$ & $7.43 \pm 2.34$ & $\mathrm{~F}=4.457$ & 0.017 \\
\hline
\end{tabular}

JOA, Japanese Orthopedic Association; MRI, magentic resonance imaging; EMG, electromyography.

Table V. Gender and cord compression ratio in each MRI/EMG patient type (number of patients).

\begin{tabular}{|c|c|c|c|c|c|c|}
\hline \multirow[b]{2}{*}{ Factor } & \multicolumn{4}{|c|}{ MRI/EMG } & \multirow[b]{2}{*}{ Test statistic } & \multirow[b]{2}{*}{ P-value } \\
\hline & $-/-$ & $+/-$ & $-/+$ & $+/+$ & & \\
\hline Gender & & & & & $\chi^{2}=0.540$ & 0.910 \\
\hline Male & 23 & 9 & 7 & 17 & & \\
\hline Female & 13 & 7 & 6 & 12 & & \\
\hline Cord compression ratio & & & & & $\chi^{2}=1.550$ & 0.671 \\
\hline$<1 / 3$ & 15 & 9 & 6 & 11 & & \\
\hline $1 / 3-1 / 2$ & 12 & 4 & 4 & 9 & & \\
\hline$>1 / 2$ & 9 & 3 & 3 & 9 & & \\
\hline
\end{tabular}

MRI, magnetic resonance imaging; EMG, electromyography.

Clinical data. Deformity of the spinal cord was expressed by the ratio of the sagittal diameter divided by the transverse diameter of the spinal cord observed on the T1-weighted transverse images (cord compression ratio = sagittal diameter/transverse diameter). The cord compression ratio was evaluated according to the method previously presented by Nagata et al $(<1 / 3,1 / 3-1 / 2$ and $>1 / 2)(10)$.

The radiological findings of all patients were evaluated and recorded in detail. The clinical parameters, including age, gender and duration of symptoms, were also recorded.

Statistical analysis. SPSS 13.0 software (Chicago, IL, USA) was used for statistical analysis. Values are presented as the mean \pm standard deviation (SD). A non-parametric analysis using the Kruskal-Wallis $\mathrm{H}$ test was used to analyze the differences among the four classification types, while the Wilcoxon test was used to analyze the differences between two types. The association between the four types and the other variables was assessed by $\chi^{2}$ and $\mathrm{F}$ tests. $\mathrm{P}<0.05$ was considered to indicate a statistically significant difference.

\section{Results}

The mean follow-up period among the patients was 23 months (range, 12-36 months) subsequent to surgery. No patients required additional cervical decompressive surgery due to restenosis or residual stenosis. Pre-operative MRI showed an alteration of the intramedullary signal on the T2-weighted MR images in 45 of 94 cases (48\%). EMG was abnormal in 42 of 94 cases $(45 \%)$. The results revealed that 36 cases $(38 \%)$ were classified as Type I, $16(17 \%)$ as Type II, 13 (14\%) as Type III and $29(31 \%)$ as Type IV.

For the pre-operative CSM disability grading, 17 cases (18\%) were categorized as grade 0,19 as grade $1(20 \%), 17$ as grade $2(18 \%), 16$ as grade $3(17 \%), 14$ as grade $4(15 \%)$ and 11 as grade 5 (12\%). The Kruskal-Wallis $\mathrm{H}$ test showed a significant difference in the disability grading between the four study groups $(\mathrm{Hc}=9.000, \mathrm{P}<0.05$; Table II). The surgical outcome was classified as excellent in 41 patients, good in 22 patients, fair in 20 patients and poor in 11 patients. The Kruskal-Wallis $\mathrm{H}$ test showed a significant difference in the recovery ratio between the classifications $(\mathrm{Hc}=27.46, \mathrm{P}<0.05)$. The Wilcoxon test showed that the surgical outcome was best in patients of Type I and worst in those of Type IV. There was no significant difference in the recovery ratio between Types II and III (Table III).

The analysis of variance for the factors of age $(\mathrm{P}=0.131)$, disease duration $(\mathrm{P}=0.004)$ and pre-operative JOA score $(\mathrm{P}=0.017)$ among the four types showed significant differences. The Student-Newman-Keuls (SNK) test analyzed the disease duration and showed significant differences between type I and II or III $(\mathrm{P}<0.05)$, type IV and II or III $(\mathrm{P}<0.05)$ and type I and IV $(\mathrm{P}<0.05)$. However, types II and III showed no significant difference $(\mathrm{P}>0.05)$. The SNK test analyzed the pre-operative JOA score and showed the same statistical results (Table IV). 
The Pearson $\chi^{2}$ test performed a gender anaysis between the four types and showed no significant differences $(\mathrm{P}=0.910)$. The Kruskal-Wallis $\mathrm{H}$ test analyzed the cord compression ratio and also showed no significant differences between the four types $(\mathrm{P}=0.671$; Table $\mathrm{V})$.

\section{Discussion}

An ISI on the T2-weighted MR images is often observed in patients with CSM and therefore the relevance of a pre-operative ISI on T2-weighted MR imaging has been extensively investigated. A number of authors have performed a series of studies on the association between ISI and surgical outcomes. Certain authors reported that patients with ISI have poor prognosis following surgery $(11,12)$, particularly those with high signal intensity ratios in the T2-weighted MR images $(13,14)$. Other studies have suggested that an ISI did not predict a poor surgical outcome in CSM $(3,15)$. In studies of histopathological examination and MRI, ISI without signal changes on the T1-weighted images appeared non-specifically in patients with mild lesion alterations, including the loss of nerve cells, gliosis, edema in the gray matter, Wallerian degeneration, demyelination and edema in the white matter (1). al-Mefty et al reported that intramedullary changes of the spinal cord observed using MRI coincided with pathological changes in the gray matter of the spinal cord, which included the loss of motor neurons, necrosis and cavitation in experimental chronic compressive myelopathies of canines (16). Therefore, the present study suggested that the signal intensity changes in MRI were able to reflect the condition of the spinal cord, but that use of only an ISI on the T2-weighted images may not exactly reflect the real neural situations of the CSM patients, and it would be more objective to combine it with the EMG examination.

Due to the compression of intra-spinal protuberances, CSM may lead to devastating and crippling neurological deficits, which are mostly detected by EMG through the abnormal stimulation of the nerve fibres travelling along the motor or sensory fascicles (10). EMG is useful for evaluating any root involvement and may be required for the exclusion of differentials, including amyotrophic lateral sclerosis and even subacute combined degeneration of the spinal cord $(4,17)$ prior to the diagnosis of CSM. In quantitative analysis, MUAP duration is the most robust feature used to differentiate between myogenic and neurogenic conditions (18). It is usual to encounter enlarged MUAPs in patients with neurogenic diseases (5), including pathological changes in the gray matter of the spinal cord. In chronic compressive myelopathy, the pathological changes in the gray matter of the spinal cord include loss of motor neurons, necrosis and cavitation $(1,16,19)$. Therefore, EMG may potentially play specific roles during the course of CSM management (4).

In the present study, there was a significant association between ISI and EMG. In the 45 patients with an ISI on the T2-weighted MR images, 29 (64.44\%) had corresponding enlarged MUAPs. As ISIs and enlarged MUAPs are able to reflect the pathological changes in the gray matter of the spinal cord, the two examinations were applied together to differentiate between the neural situations in the CSM patients. Based on the MRI/EMG classification system the patients were divided into four types: Type I, MRI/EMG (-/-); Type II, MRI/EMG (+/-); Type III, MRI/EMG (-/+); and Type IV, MRI/EMG (+/+). The patients underwent surgical intervention by anterior, posterior and posterior-anterior united decompression based on their cord compression ratio and other factors. Until the final follow-up, a different recovery ratio was observed between the four study groups. The surgical outcome was best in Type I and worst in Type IV patients, while the outcome for patients of Types II and III were observed in-between. The results revealed that the patients without ISIs or enlarged MUAPs were able to obtain a good prognosis.

Ramanauskas et al divided myelomalacia into three stages: early, intermediate and late (20). A change in the signal intensity of the spinal cord observed on MRI reflected cord edema in the early stage and cystic necrosis of the central gray matter following prolonged cord edema in the late stage. Zhang et al quantized the signal intensity ratio of the T2-weighted MRI and identified that an increase in signal intensity ratio was associated with older patients, longer disease duration and a trend for a reduced recovery rate (13). In the present study, relative index also had the tendency to have more severe symptoms and a worse prognosis. Type IV patients had a lower pre-operative JOA score, a longer disease duration and most significant of all, a worse post-operative recovery rate than patients of the other three types. These factors have previously been reported to affect the surgical outcome in patients with CSM (21-23).

In summary, these results indicate that there is a distinct correlation between EMG combined with MRI and the rate of clinical improvement in CSM patients. Patients who have a negative EMG and who lack an ISI tend to have mild symptoms, a short disease duration and experience a good prognosis. The grading system based on the EMG and MRI results is extremely thorough, appears to be objective for each observer and is beneficial for forming a prognosis of the surgical outcome of CSM.

\section{References}

1. Ohshio I, Hatayama A, Kaneda K, Takahara M and Nagashima K: Correlation between histopathologic features and magnetic resonance images of spinal cord lesions. Spine (Phila Pa 1976) 18: 1140-1149, 1993.

2. Yukawa Y, Kato F, Yoshihara H, Yanase M and Ito K: MR T2 image classification in cervical compression myelopathy: predictor of surgical outcomes. Spine (Phila Pa 1976) 32: 1675-1679, 2007.

3. Shen HX, Li L, Yang ZG and Hou TS: Position of increased signal intensity in the spinal cord on MR images: does it predict the outcome of cervical spondylotic myelopathy? Chin Med J (Engl) 122: 1418-1422, 2009.

4. Lo YL: How has electrophysiology changed the management of cervical spondylotic myelopathy? Eur J Neurol 15: 781-786, 2008.

5. Barkhaus PE and Nandedkar SD: On the selection of concentric needle electromyogram motor unit action potentials: is the rise time criterion too restrictive? Muscle Nerve 19: 1554-1560, 1996.

6. Bednarik J, Kadanka Z, Dusek L, et al: Presymptomatic spondylotic cervical myelopathy: an updated predictive model. Eur Spine J 17: 421-431, 2008.

7. Nurick S: The pathogenesis of the spinal cord disorder associated with cervical spondylosis. Brain 95: 87-100, 1972.

8. Naderi S, Ozgen S, Pamir MN, Ozek MM and Erzen C: Cervical spondylotic myelopathy: surgical results and factors affecting prognosis. Neurosurgery 43: 43-50, 1998. 
9. Kiris $\mathrm{T}$ and Kilinçer $\mathrm{C}$ : Cervical spondylotic myelopathy treated by oblique corpectomy: a prospective study. Neurosurgery 62 : 674-682, 2008

10. Nagata K, Kiyonaga K, Ohashi T, Sagara M, Miyazaki S and Inoue A: Clinical value of magnetic resonance imaging for cervical myelopathy. Spine (Phila Pa 1976) 15: 1088-1096, 1990.

11. Okada Y, Ikata T, Yamada H, Sakamoto R and Katoh S: Magnetic resonance imaging study on the results of surgery for cervical compression myelopathy. Spine (Phila Pa 1976) 18: 2024-2029, 1993.

12. Suri A, Chabbra RP, Mehta VS, Gaikwad S and Pandey RM: Effect of intramedullary signal changes on the surgical outcome of patients with cervical spondylotic myelopathy. Spine J 3: 33-45, 2003.

13. Zhang YZ, Shen Y, Wang LF, Ding WY, Xu JX and He J: Magnetic resonance T2 image signal intensity ratio and clinical manifestation predict prognosis after surgical intervention for cervical spondylotic myelopathy. Spine (Phila Pa 1976) 35: E396-E399, 2010.

14. Zhang P, Shen Y, Zhang YZ, Ding WY and Wang LF: Significance of increased signal intensity on MRI in prognosis after surgical intervention for cervical spondylotic myelopathy. J Clin Neurosci 18: 1080-1083, 2011.

15. Mastronardi L, Elsawaf A, Roperto R, et al: Prognostic relevance of the postoperative evolution of intramedullary spinal cord changes in signal intensity on magnetic resonance imaging after anterior decompression for cervical spondylotic myelopathy. J Neurosurg Spine 7: 615-622, 2007.

16. al-Mefty O, Harkey HL, Marawi I, et al: Experimental chronic compressive cervical myelopathy. J Neurosurg 79: 550-561, 1993
17. Lo YL, Chan LL, Leoh T, et al: Diagnostic utility of F waves in cervical radiculopathy: electrophysiological and magnetic resonance imaging correlation. Clin Neurol Neurosurg 110: 58-61, 2008.

18. Stewart CR, Nandedkar SD, Massey JM, Gilchrist JM, Barkhaus PE and Sanders DB: Evaluation of an automatic method of measuring features of motor unit action potentials. Muscle Nerve 12: 141-148, 1989.

19. Ito T, Oyanagi K, Takahashi H, Takahashi HE and Ikuta F: Cervical spondylotic myelopathy. Clinicopathologic study on the progression pattern and thin myelinated fibers of the lesions of seven patients examined during complete autopsy. Spine (Phila $\mathrm{Pa}$ 1976) 21: 827-833, 1996

20. Ramanauskas WL, Wilner HI, Metes JJ, Lazo A and Kelly JK: MR imaging of compressive myelomalacia. J Comput Assist Tomogr 13: 399-404, 1989.

21. Morio Y, Teshima R, Nagashima H, Nawata K, Yamasaki D and Nanjo Y: Correlation between operative outcomes of cervical compression myelopathy and mri of the spinal cord. Spine (Phila Pa 1976) 26: 1238-1245, 2001.

22. Chen CJ,Lyu RK,Lee ST, Wong YC and Wang LJ: Intramedullary high signal intensity on T2-weighted MR images in cervical spondylotic myelopathy: prediction of prognosis with type of intensity. Radiology 221: 789-794, 2001

23. Yukawa Y, Kato F, Ito K, et al: Postoperative changes in spinal cord signal intensity in patients with cervical compression myelopathy: comparison between preoperative and postoperative magnetic resonance images. J Neurosurg Spine 8: 524-528, 2008. 